\title{
Potential of the Akt inhibitor LY294005 to antagonize the efficacy of Cisplatin against HCT116 tumor cells in a DNA mismatch repair-dependent manner
}

\author{
ANDRE FEDIER $^{1}$, RUEDIGER ERDMANN $^{1}$, TENI BOULIKAS $^{2}$ and DANIEL FINK ${ }^{1}$ \\ ${ }^{1}$ Department of Gynecology, University Hospital Zurich, Frauenklinikstrasse 10, CH-8091 Zurich, Switzerland; \\ ${ }^{2}$ Regulon, Inc., 715 North Shoreline Boulevard, Mountain View, CA 94043, USA
}

Received March 16, 2006; Accepted May 12, 2006

\begin{abstract}
Human colorectal adenocarcinoma sublines either deficient (HCT116+ch2) or proficient $(\mathrm{HCT} 116+\mathrm{ch} 3)$ in the function of MLH1, one of five proteins crucial to DNA mismatch repair (MMR), were used to investigate whether the Akt-specific inhibitor LY294005 could not only increase the efficacy of platinum drugs in HCT116 cells in general but also increase the efficacy of the cisplatinum compounds Cisplatin and Lipoplatin specifically in MLH1-deficient, Cisplatin- and Lipoplatin-resistant HCT116 cells. We report that, under the conditions it increased the efficacy of Docetaxel and did not affect that of 6-thioguanine, LY294005 decreased the sensitivity of both sublines to Cisplatin, Lipoplatin, Oxaliplatin, and Lipoxal. Notably, the LY294005-imposed decrease was significantly higher in the MLH1-proficient than in the MLH1-deficient subline with Cisplatin and Lipoplatin, whereas it was nearly the same in both sublines with Oxaliplatin and Lipoxal. These LY294005-imposed changes in drug sensitivity, i.e. increase with Docetaxel and decreases with platinum compounds, were not associated with the concomitant abrogation in the levels of phospho$\mathrm{Akt}^{\text {ser473}}$. Analogous changes in drug sensitivity were also observed with the PI3-kinase inhibitor LY294002, but these changes were associated with complete abrogation of phospho-Akt ${ }^{\mathrm{ser} 473}$. These observations suggest a possible relationship between MMR-mediated cisplatinum DNA damage signaling and the Akt signaling pathway, e.g. a common target for both pathways. A possibly novel property of Akt in aggravating drug sensitivity may also be proposed.
\end{abstract}

Correspondence to: Dr André Fedier, Department of Gynecology, University Hospital of Zurich, Frauenklinikstrasse 10, CH-8091 Zurich, Switzerland

E-mail: andre.fedier@usz.ch

Key words: Akt, Akt inhibitor LY294005, DNA mismatch repair, platinum drug resistance

\section{Introduction}

DNA mismatch repair (MMR) is a post-replicative repair machinery that recognizes and corrects DNA biosynthetic errors occurring spontaneously during replication. It therefore contributes to the maintenance of genome integrity and to the prevention of carcinogenesis (1-5). MMR is also linked to the control of cell cycle check-points and apoptosis $(6,7)$. Many studies including ours have shown that loss of MMR function is associated with resistance to the cytotoxic effect of several types of DNA damaging agents such as alkylating agents, platinum compounds, mercaptopurines, and anthracyclines (8-13). Clinical studies support the role of MMRdeficiency in drug resistance and its biological relevance $(14,15)$.

A recent study has shown that Akt overexpression fails to activate the $\mathrm{G}_{2} / \mathrm{M}$ check-point in MMR-proficient tumor cells and hence mimics the phenotype seen for MMR-deficient tumor cells (16). Akt (also referred to as protein kinase B, $\mathrm{PKB}$ ) is activated by the binding of growth factors to receptor tyrosine kinases and the activation of the phosphatidylinositol 3-kinase (PI3K). PI3K catalyzes the conversion of $\mathrm{PIP}_{2}$ lipids into $\mathrm{PIP}_{3}$ lipids. $\mathrm{PIP}_{3}$ serves to anchor Akt to the plasma membrane, where Akt is phosphorylated at threonine 308 and serine 473 of the membrane-bound protein and fully activated by 3 -phosphoinositide-dependent kinases (PDKs). Akt, itself a serine/threonine kinase, is then re-distributed to the cytoplasm and nucleus and phosphorylates a variety of substrates (17-19). The main role attributed to Akt signaling is to facilitate cell growth and proliferation $(20,21)$ and to block cell death $(22,23)$. The Akt signaling pathway is up-regulated in cancers, is critical for tumorigenesis and chemoresistance, and is therefore a critical target for cancer intervention and for overcoming drug resistance $(24,25)$. Studies have shown that down-regulation or target-specific inhibition of Akt signaling increases sensitivity of tumor cells to irradiation and to chemotherapeutic agents (26-33).

Akt might also be involved in responding to genotoxic stress. ATM phosphorylates Akt at serine 473 following irradiation (34), and ATM, CHK1/2, and BRCA1 have been shown to be, either directly or indirectly, substrates to phosphorylation by Akt $(20,21,35,36)$. BRCA1 and ATM have been found to associate with MMR proteins in the BASC 
super-complex (37). However, the nature of putative common targets for MMR proteins and Akt (16) is unclear as is the issue whether Akt signaling contributes to drug resistance in MMR-deficient cells. We hypothesized that the survival property of Akt is repressed by functional MMR, whereas this repression is absent in MMR-deficient cells, rendering them drug resistant. We further hypothesized that inhibition of Akt by LY294005 (27) re-introduces in MMR-deficient cells the ability to repress Akt signaling, rendering them chemosensitive. Our hypothesis was tested with the MMRdependent drugs Cisplatin, a widely used and effective agent for the treatment of human malignancies, Lipoplatin, a recently developed liposome-encapsulated Cisplatin formulation showing high anticancer activity but low adverse side-effects often seen for Cisplatin (38), and 6-thioguanine.

Our observations reveal the potential of LY294005 to antagonize the efficacy of platinum drugs against HCT116 tumor cells. Although not conclusive, a possible property of Akt may be suggested that aggravates the cytotoxic effect of platinum compounds and that seems to be, at least for cisplatinum drugs, modulated by the integrity of MMR function.

\section{Materials and methods}

Cell lines. A matched pair of a MLH1-deficient human colorectal adenocarcinoma subline (designated HCT116+ch2) and its MLH1-proficient counterpart (designated HCT116+ch3) was used. They are derived from the human colorectal adenocarcinoma cell line HCT116 containing a hemizygous mutation in $M L H 1$ resulting in a truncated, non-functional protein (American Type Culture Collection; ATCC CCL 247). The HCT116+ch3 subline is complemented with chromosome 3 carrying the wild-type gene for $h M L H 1$ and is competent in MMR function. The HCT116+ch2 subline, for reasons of chromosome balance, is complemented with chromosome 2 and is MMR incompetent. Both sublines were maintained in Iscove's modified Dulbecco's medium (Invitrogen, Basel, Switzerland) supplemented with $10 \%$ heat-inactivated fetal bovine serum (Oxoid, Basel, Switzerland) and geneticin (400 $\mu \mathrm{g} / \mathrm{ml})$ (Invitrogen). It is generally acknowledged that the chromosome complementation does not spoil the effects of DNA mismatch repair on drug sensitivity, although the extent of possible effects from the introduction of an extra chromosome is not fully clear. The presence or absence of MLH1 protein in the respective sublines is routinely checked by immunoblotting as is the response of the two sublines to the alkylating agent $N$-methyl- $N$ '-nitro- $N$-nitrosoguanidine (not shown) to which the MLH1-deficient HCT116+ch2 subline is resistant (8). The growth rates of the two sublines, calculated from the doubling times from one passage to the subsequent and averaged for a period of two months, were comparable (21.4 $\pm 1.1 \mathrm{~h}$ for HCT116+ch3; 21.6 $\pm 0.9 \mathrm{~h}$ for HCT116+ch2). When seeded sparsely on tissue culture plates, the cell lines form well-defined individual colonies.

Drugs and chemicals. Cisplatin and 6-thioguanine were purchased (Sigma, Buchs, Switzerland), and Oxaliplatin (Sanofi-Synthelabo, Meyrin, Switzerland) was a generous gift. Stock solutions were prepared in $0.9 \% \mathrm{NaCl}$ and stored at $-20^{\circ} \mathrm{C}$. Lipoplatin and Lipoxal, the liposome-encapsulated formulations of Cisplatin and Oxaliplatin, respectively, were synthesized and provided by Regulon, Inc. (Mountain View, CA, USA) and stored at $4^{\circ} \mathrm{C}$. Docetaxel (Aventis, Zurich, Switzerland) was dissolved in ethanol and stored at $-20^{\circ} \mathrm{C}$. The compound 1L-6-hydroxymethyl-chiro-inositol 2-(R)-2O-methyl-3-O-octadecylcarbonate (hereafter referred to as LY294005) was purchased from Juro Supply (Lucerne, Switzerland). This compound is a recently developed 3(hydroxymethyl)-bearing phosphatidylinositol ether lipid analogue that selectively inhibits PI3K/Akt activity and growth of tumor cell lines (27). The PI3-kinase inhibitor LY294002 was purchased (Sigma).

Clonogenic assay. The sensitivity of the cells to treatments was determined by the clonogenic assay. Sensitivity to single drug treatment was compared with that of the combination of the drug with LY294005 or LY294002. In a typical setting, 300 cells in medium were plated onto $35-\mathrm{mm}$ cell culture dishes, followed by the addition of the inhibitor after $24 \mathrm{~h}$. Where appropriate, the drugs were added at various concentrations $6 \mathrm{~h}$ later, followed by incubation for another $20 \mathrm{~h}$ at $37^{\circ} \mathrm{C}$ in a humidified atmosphere containing $5 \%$ carbon dioxide. Then the medium was exchanged, followed by incubation for another 6 days to allow colony formation. This regimen, i.e. pre-treatment of the cells with the inhibitor prior to drug exposure, was chosen in order to pre-condition the cells before the drugs initiate damage response pathways. Cells were then fixed with $25 \%$ acetic acid in ethanol and stained with Giemsa. Colonies of at least 50 cells were scored visually. Each experiment was performed a minimum of three times using duplicate cultures for each drug concentration. The relative colony formation (\% clonogenic survival) was plotted against the treatments.

Immunoblot analysis. Immunoblot analysis was performed to determine treatment-induced alterations in the level of phosphorylated Akt at serine 473. Cells $(500,000)$ grown to $80 \%$ confluence in 60-mm tissue culture dishes were further incubated without or with LY294005 $(10 \mu \mathrm{M})$ or LY294002 $(20 \mu \mathrm{M})$ for another $6 \mathrm{~h}$. Where appropriate, Cisplatin, Oxaliplatin, or Docetaxel was added to the cultures, followed by further incubation of the cultures for $18 \mathrm{~h}$. Some cultures were also treated with $20 \mathrm{ng} / \mathrm{ml}$ insulin growth factor IGF. Cells were collected, washed in PBS, and prepared for immunoblot analysis following standard protocols. The lysis buffer contained DTT $(1 \mathrm{mM})$, the protease inhibitors aprotinin $(1 \mu \mathrm{g} / \mathrm{ml})$, PMSF $(1 \mathrm{mM})$, benzamidin $(1 \mathrm{mM})$, and the phosphatase inhibitors $\beta$-glycerol-phosphate $(20 \mathrm{mM})$, $\mathrm{Na}_{3} \mathrm{VO}_{4}(1 \mathrm{mM})$, and $\mathrm{NaF}(25 \mathrm{mM})$. Protein $(20 \mu \mathrm{g})$ was separated using $10 \%$ SDS-PAGE, followed by blotting onto a Protran nitrocellulose membrane (Schleicher and Schuell BioScience Inc., Keene, NH). Proteins were complexed using the anti-Akt total (Cell Signalling 9272, BioConcept, Allschwil, Switzerland) and anti-phospho-Akt ${ }^{\mathrm{ser}} 473$ (Cell Signalling 9271) primary antibodies, and the respective horseradish peroxideconjugated secondary antibody (Cell Signalling 7074). ß-actin, detected by monoclonal anti-ß-actin (A5441, Sigma) and horseradish peroxidase-conjugated anti-mouse antibody (M15345; Transduction Laboratories, Lexington, KY), served 
A

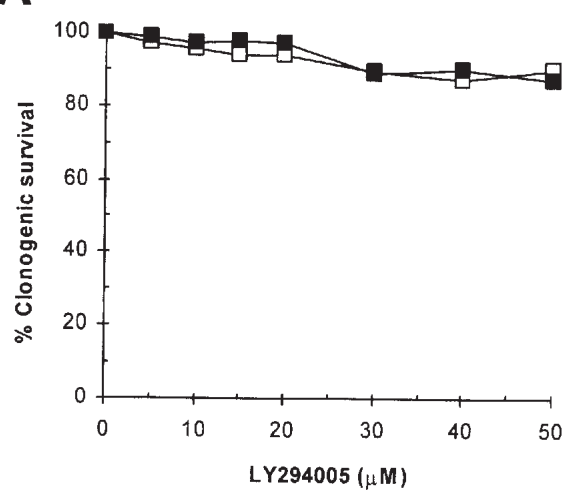

B

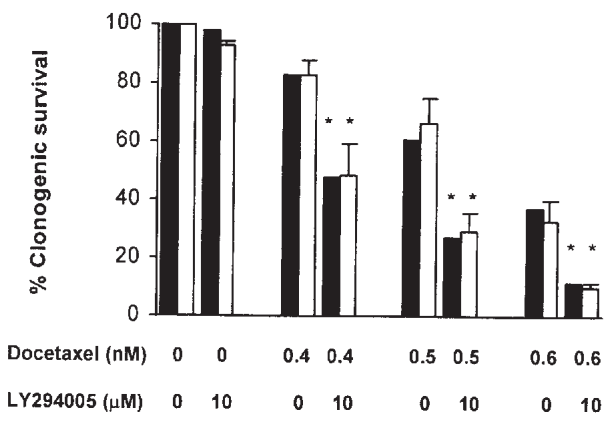

Figure 1. The sensitivity of cells to LY294005 itself and the effect of LY294005 on sensitivity to Docetaxel (\% of clonogenic survival). (A) MLH1-proficient HCT116+ch3 (ם) and MLH1-deficient HCT116+ch2 ( $\square$ ) human colorectal adenocarcinoma cells were grown in the absence or presence of various concentrations of LY294005 for $24 \mathrm{~h}$. After medium exchange, cells were grown for another 6 days and then colonies were stained with Giemsa and counted. Data points are the mean of two independent experiments performed in duplicate cultures. (B) MLH1proficient HCT116+ch3 (ם) and MLH1-deficient HCT116+ch2 ( $\square$ ) cells were grown in the absence or presence of LY294005 $(10 \mu \mathrm{M})$ and without or with various concentrations of Docetaxel. LY294005 was added $6 \mathrm{~h}$ prior to the addition of Docetaxel. The medium was replaced $24 \mathrm{~h}$ post the addition of Docetaxel, the cells were grown for another 6 days to allow colony formation. Data points are the mean \pm SEM of three independent experiments performed in duplicate cultures. ${ }^{*} \mathrm{p}<0.05$ compared to cultures without LY294005.

as the sample loading control. The complexes were visualized by enhanced chemiluninescence (Amersham Pharmacia Biotech, Buckinghamshire, UK) and autoradiography.

Statistical analysis. The mean \pm SD or the mean \pm SEM were calculated for all data sets (paired, two-tailed Student's t-test). A $p<0.05$ was considered statistically significant.

\section{Results}

Effect of LY294005 on the efficacy of Docetaxel. The Akt inhibitor LY294005 has recently been shown to prevent phosphorylation of Akt by the 3-phosphoinositide-dependent kinases and to selectively ( $\mathrm{IC}_{50}: 5 \mu \mathrm{M}$ ) block Akt activity (27). Here we show that concentrations of LY294005 as high as $50 \mu \mathrm{M}$ reduced the clonogenic potential by no more than $15 \%$ (Fig. 1A). We determined whether a LY294005 concentration of $10 \mu \mathrm{M}$, which by itself did not affect the clonogenic potential of HCT116 cells, was high enough to chemosensitize HCT116 tumor cells to Docetaxel, a micro-
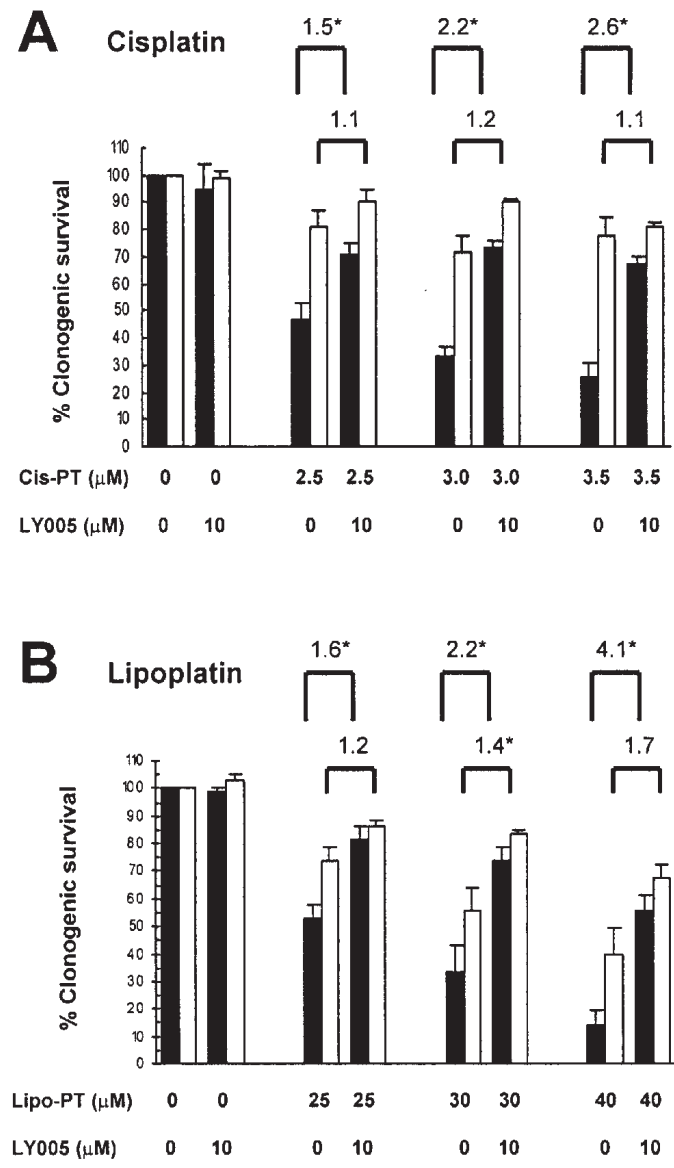

Figure 2. Effect of LY294005 on the sensitivity (\% clonogenic survival) of cells to cisplatinum drugs. MLH1-proficient HCT116+ch3 (ם) and MLH1-deficient HCT116+ch2 $\square$ ) cells were grown in absence or presence of $10 \mu \mathrm{M}$ LY294005 (LY005) and without or with various concentrations of (A) Cisplatin (Cis-PT) or (B) Lipoplatin (Lipo-PT). LY294005 was added $6 \mathrm{~h}$ prior to the addition of the platinum drugs. After $24 \mathrm{~h}$ the drug-containing medium was replaced by drug-free medium, and the cells were grown for another 6 days. Colonies were stained with Giemsa and counted. Data points are the mean \pm SEM of at least three independent experiments performed in duplicate cultures. The figures atop the brackets refer to the values for the fold decrease in sensitivity (increase in \% clonogenic survival) imposed by LY294005. ${ }^{*} \mathrm{p}<0.05$ compared to cultures without LY294005.

tubule poison of the family of taxanes not known to interact with DNA or to require MMR function for its cytotoxic effect. The results show (Fig. 1B) that pre-treatment with $10 \mu \mathrm{M}$ LY294005 produced, irrespective of the MLH1 status of the subline, a significant increase in the sensitivity (i.e. decrease in clonogenic survival) to Docetaxel, ranging from 1.7 -fold $(0.4 \mathrm{nM})$ to 3.3 -fold $(0.6 \mathrm{nM})$. A concentration of $10 \mu \mathrm{M}$ LY294005 was thus sufficient to increase the efficacy of Docetaxel against HCT116 cells and was used in all following experiments.

MLH1-dependent antagonistic effect of LY294005 on the efficacy of cisplatinum drugs. With regard to our hypothesis and to our observation with Docetaxel, we determined whether $10 \mu \mathrm{M}$ LY294005 not only increased the efficacy of cisplatinum drugs (Cisplatin and Lipoplatin) against HCT116 cells in general but also did so with a preference for the MLH1-deficient HCT116+ch2 subline. With Cisplatin, we found that for both sublines pre-treatment with LY294005 
resulted in a decrease rather than the expected increase in the sensitivity of both sublines to this compound (Fig. 2A). Notably, the extent of this decrease in sensitivity to Cisplatin was higher for the MLH1-proficient subline than for the MLH1-deficient subline: the LY294005-imposed decreases in sensitivity (i.e. increases in the clonogenic potential) for the MLH1-proficient subline were 1.5-fold (2.5 $\mu \mathrm{M})$, 2.2-fold $(3.0 \mu \mathrm{M})$, and 2.6 -fold $(3.5 \mu \mathrm{M})$, and were all statistically significant $(\mathrm{p}<0.05)$. For the MLH1-deficient subline, the respective values were 1.1-, 1.2-, and 1.1-fold, and were not statistically significant. This showed that this LY294005imposed effect occurred with a reproducible preference (1.4-, 1.8-, and 2.4-fold, respectively) for the MLH1-proficient subline. Likewise, LY294005 also decreased the sensitivity to Lipoplatin (Fig. 2B). These decreases were 1.6-fold (25 $\mu \mathrm{M}$ Lipoplatin), 2.2-fold (30 $\mu \mathrm{M})$, and 4.1-fold (40 $\mu \mathrm{M})$ for the MLH1-proficient subline, and were all statistically significant $(\mathrm{p}<0.05)$. Those for the MLH1-deficient subline were 1.2-, 1.4- (statistically significant), and 1.7-fold, respectively. This showed that the decreases occurred with a preference for the MLH1-proficient subline (1.3-, 1.7-, and 2.4-fold, respectively). Thus $10 \mu \mathrm{M}$ LY294005 antagonized the efficacy of Cisplatin and Lipoplatin against HCT116 cells with a reproducible preference for MLH1-proficient cells and therefore in an MLH1dependent manner.

MLH1-independent antagonistic effect of LY294005 on the efficacy of oxaliplatinum drugs. Our observations for the platinum compounds indicated that LY294005 may modulate the platinum drug sensitivity of HCT116 cells in a MLH1dependent manner. As Oxaliplatin had previously been shown to exert its cytotoxic effect independently of MMR (9), we expected that, in this case, the effect of LY294005 on the efficacy of Oxaliplatin would therefore be similar for both sublines. Indeed, pre-treatment with $10 \mu \mathrm{M}$ LY294005 resulted in a statistically significant decrease in the sensitivity to Oxaliplatin, the extent of which was comparable in both sublines (Fig. 3A). The LY294005-imposed decreases were 1.6-fold for the MLH1-proficient subline and 1.5-fold for the MLH1-deficient subline (3 $\mu \mathrm{M}$ Oxaliplatin, 1.1-fold difference), 2.5- and 2.3-fold (4 $\mu \mathrm{M}, 1.1$-fold), and 6.4- and 5.4-fold (5 $\mu \mathrm{M}, 1.2$-fold), respectively, and were all statistically significant $(\mathrm{p}<0.05)$. Similar observations also were made with Lipoxal (Fig. 3B). With this compound the LY294005imposed decreases in the sensitivity of the sublines were 1.3-fold for the MLH1-proficient subline and 1.3-fold for the MLH1-deficient subline (3 $\mu \mathrm{M}$ Lipoxal), 1.9- and 2.0-fold (4 $\mu \mathrm{M})$, and 2.7- and 2.8-fold (5 $\mu \mathrm{M})$, respectively. Like with Oxaliplatin, these LY294005-imposed decreases in sensitivity to Lipoxal were all statistically significant and their extents were nearly the same for both sublines. LY294005 $(10 \mu \mathrm{M})$ thus antagonized the efficacy of Oxaliplatin and Lipoxal against HCT116 cells without a preference for either the one or the other subline and therefore in an MLH1-independent manner.

Effect of LY294005 on the efficacy of 6-thioguanine. Our observations with cisplatinum drugs raised the question as to whether the MLH1-dependent modulating effect of LY294005 seen for these drugs was also seen with the antimetabolite 6-thioguanine, the cytotoxic effect of which is dependent on
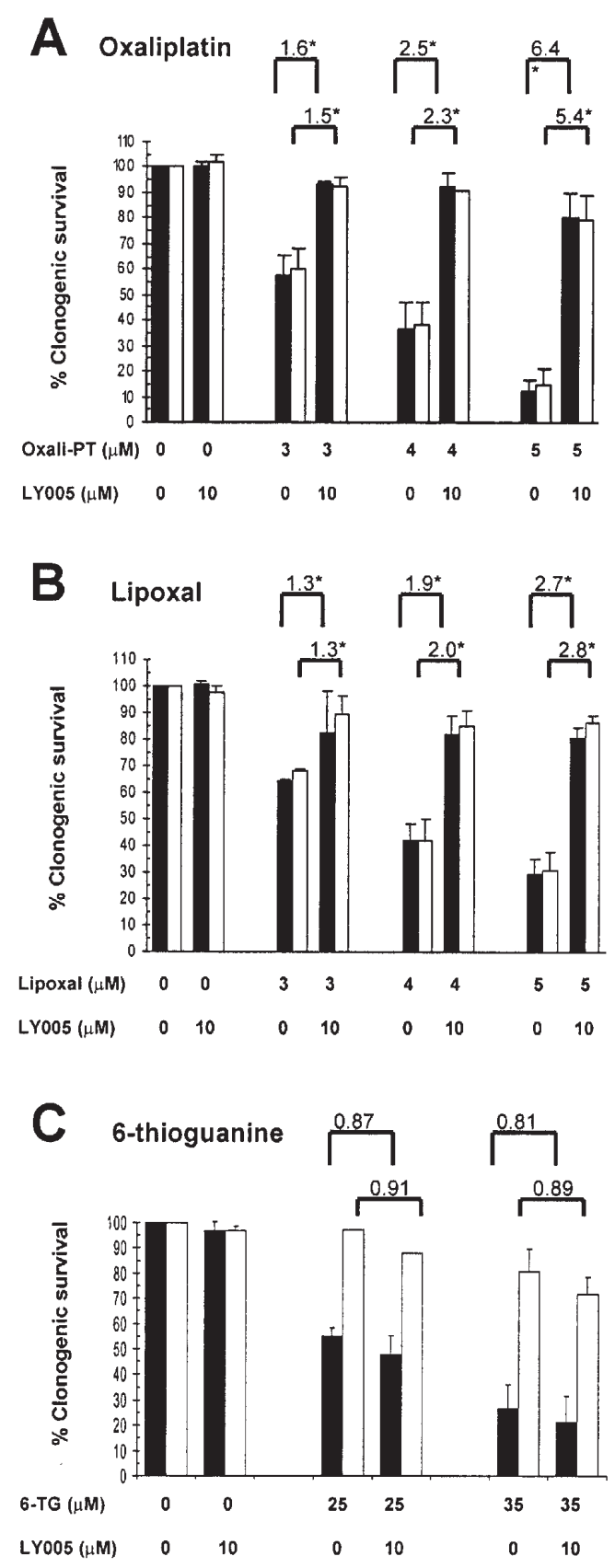

Figure 3. Effect of LY294005 on the sensitivity (\% clonogenic survival) of cells to oxaliplatinum compounds and the antimetabolite 6-thioguanine. MLH1-proficient HCT116+ch3 (ロ) and MLH1-deficient HCT116+ch2 ( $\square$ ) cells were grown in the absence or presence of $10 \mu \mathrm{M}$ LY294005 (LY005) and without or with various concentrations of (A) Oxaliplatin (Oxali-PT), (B) Lipoxal, or (C) 6-thioguanine (6-TG). LY294005 was added $6 \mathrm{~h}$ prior to the addition of the platinum drugs. The drug-containing medium was replaced by drug-free medium after $24 \mathrm{~h}$ post the platinum drug addition, followed by growth of the cells for another 6 days, staining of the colonies with Giemsa and colony counting. Data points are the mean \pm SEM of at least three independent experiments performed in duplicate cultures. The figures atop the brackets refer to the values for the fold decrease in sensitivity (increase in \% clonogenic survival) imposed by LY294005. * $\mathrm{p}<0.05$ compared to cultures without LY294005.

the presence on MLH1 (10). The data showed that $10 \mu \mathrm{M}$ LY294005 did not affect the efficacy of 6-thioguanine against both the MLH1-proficient and the MLH1-deficient sublines (Fig. 3C). Thus LY294005, in an experimental setting 

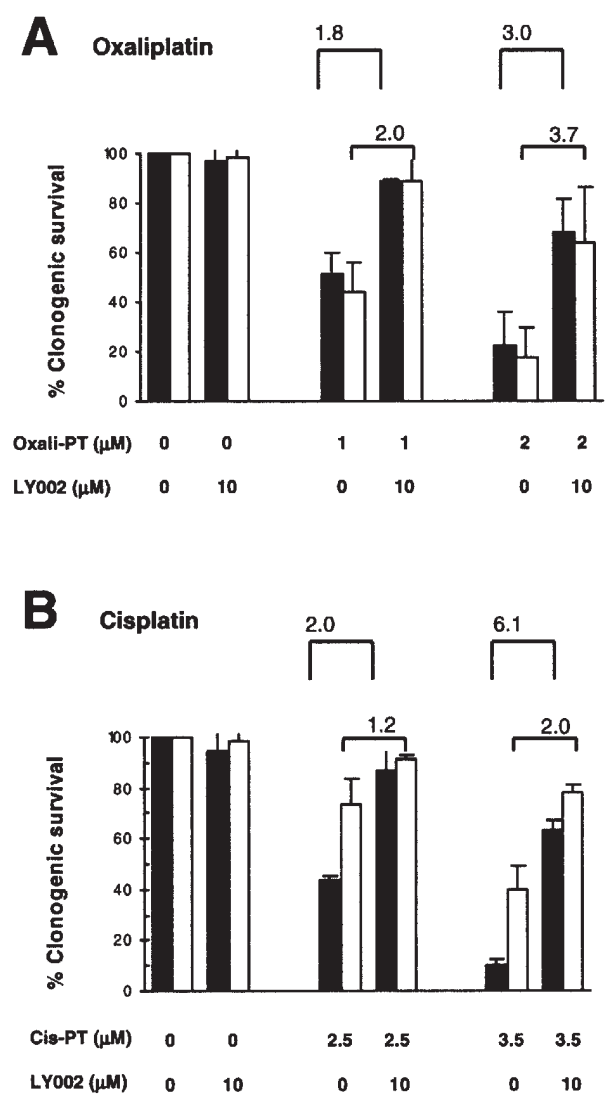

Figure 4. Effect of LY294002 on the sensitivity (\% clonogenic survival) of cells to Oxaliplatin (A) and Cisplatin (B). MLH1-proficient HCT116+ch3 (ם) and MLH1-deficient HCT116+ch2 $(\square)$ cells were grown in the absence or presence of $10 \mu \mathrm{M}$ LY294002 (LY002) and without or with various concentrations of Oxaliplatin (Oxali-PT) or Cisplatin (Cis-PT). LY294002 was added $6 \mathrm{~h}$ prior to the addition of the platinum drugs. After $24 \mathrm{~h}$ the drug-containing medium was replaced by drug-free medium and the cells were grown for another 6 days. Colonies were stained with Giemsa and counted. Data points are the mean values from two independent experiments performed in duplicate cultures. The figures atop the brackets refer to the values for the fold decrease in sensitivity (increase in \% clonogenic survival) imposed by LY294002.

analogous to that with cisplatinum drugs and Docetaxel, neither decreased nor increased the efficacy of 6-thioguanine.

Antagonistic effect of LY294002 on the efficacy of Oxaliplatin and Cisplatin. Our observations with LY294005 indicate that LY294005 can not only decrease the efficacy of platinum drugs but also modulate that of cisplatinum drugs in a manner depending on the presence or absence of MLH1 protein. We determined whether the observed antagonizing effect of LY294005 on platinum drug sensitivity was unique for the LY294005 Akt inhibitor or whether it was also seen with LY294002, an inhibitor for the PI3-kinase. As with LY294005, a decrease in the sensitivity to Oxaliplatin and Cisplatin was also observed with $10 \mu \mathrm{M}$ LY294002. With Oxaliplatin (Fig. 4A), these LY294002-imposed decreases were comparable in both sublines and therefore were not affected by the MLH1 status of the cells. But they were greater in the MLH1-proficient subline than in the MLH1deficient subline with Cisplatin (Fig. 4B): with $2.5 \mu \mathrm{M}$ Cisplatin, the LY294002-imposed increases were 2.0-fold for the MLH1-proficient subline and 1.2-fold for the MLH1- deficient subline (1.7-fold preference for the MLH1-proficient subline), and with $3.5 \mu \mathrm{M}$ Cisplatin these increases were 6.1and 2.0-fold, respectively (3.1-fold preference). The modulating effect on platinum drug sensitivity observed with LY294005 was also seen with the PI3-kinase inhibitor LY294002 and hence was not unique to the LY294005 Akt inhibitor.

Effect of LY294005 on Akt ser473 phosphorylation. The sensitizing effect of LY294005 to methylselenic acid has recently been reported to be associated with the concomitant reduction in the level of phospho-Akt $\mathrm{Ar}^{\mathrm{s} 43}$ (26). We determined whether the observed LY294005-imposed effects on drug sensitivity were accompanied by changes in the phosphorylation status of Akt. Immunoblot data (Fig. 5A) revealed that the basal level of phospho- $\mathrm{Akt}^{\mathrm{ser} 473}$ (upper band) was quite low in both sublines, as compared to the respective levels for cells stimulated with $20 \mathrm{ng} / \mathrm{ml}$ IGF. LY294005 by itself $(10 \mu \mathrm{M})$ did not affect phospho-Akt ${ }^{\text {ser473 }}$ in samples not treated with drugs (none), and likewise, LY294005 $(10 \mu \mathrm{M})$ did not abolish $\mathrm{Akt}^{\mathrm{ser} 473}$ phosphorylation in samples treated with either Docetaxel (Doce, $0.6 \mathrm{nM}$ ), Oxaliplatin (Oxali, $5 \mu \mathrm{M}$ ), or Cisplatin (Cis, $5 \mu \mathrm{M}$ ). This showed that the observed synergistic effect of LY294005 on the efficacy of Docetaxel was not paralleled by a reduction of phospho-Akt ${ }^{\mathrm{ser} 473}$. This also showed that the LY294005-imposed antagonizing effects on the efficacy of platinum drugs were not accompanied by changes in the level of phospho-Akt $\mathrm{ter}^{\mathrm{s} 43}$. These results suggested that LY294005 $(10 \mu \mathrm{M})$ was unable to abrogate $\mathrm{Akt}^{\text {ser473 }}$ phosphorylation. This was supported by the observations with LY294005 in IGF-stimulated samples (Fig. 5B), where even $25 \mu \mathrm{M}$ LY294005 did not abrogate IGFinduced Akt phosphorylation in both sublines. The results also indicated that $10 \mu \mathrm{M}$ LY294005 differentially, i.e. depending on the drug type, affected drug sensitivity without the concomitant abrogation of $\mathrm{Akt}^{\mathrm{ser} 473}$ phosphorylation. For closer examination and for comparison of the effects of LY294005 with those of LY294002, we determined the phospho-Akt ${ }^{\text {ser473 }}$ status at various time-points after treatment without or with either LY294005 or LY294002 in samples treated or not with Cisplatin or Docetaxel. With Cisplatin and with both sublines

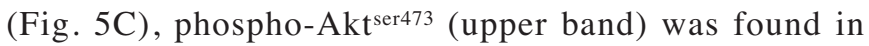
untreated control samples (lane A), in samples treated with only Cisplatin (lane F), and in those pre-treated with LY294005 without Cisplatin (lanes B and C) and with Cispaltin (lanes D and E), but not in samples pre-treated with LY294002 without (lanes $\mathrm{G}$ and $\mathrm{H}$ ) and with Cisplatin (lanes I and K). Analogous observations were made with Docetaxel (Fig. 5D). Pretreatment with LY294005 did not affect the level of phospho$\mathrm{Akt}^{\mathrm{ser} 473}$ (lanes B-D), whereas pre-treatment with LY294002 produced a complete reduction in the levels of phospho-Akt ${ }^{\mathrm{ser} 473}$ in both sublines (lanes F-H).

Thus $10 \mu \mathrm{M}$ LY294005, unlike $20 \mu \mathrm{M}$ LY294002, did not abrogate phosphorylation of $\mathrm{Akt}^{\mathrm{ser} 473}$, but nevertheless produced an increase in the efficacy of Docetaxel and a decrease in the efficacy of platinum drugs against HCT116 cells.

\section{Discussion}

Previous studies have shown the radio- and chemosensitizing effect of the target-specific down-regulation of the Akt 

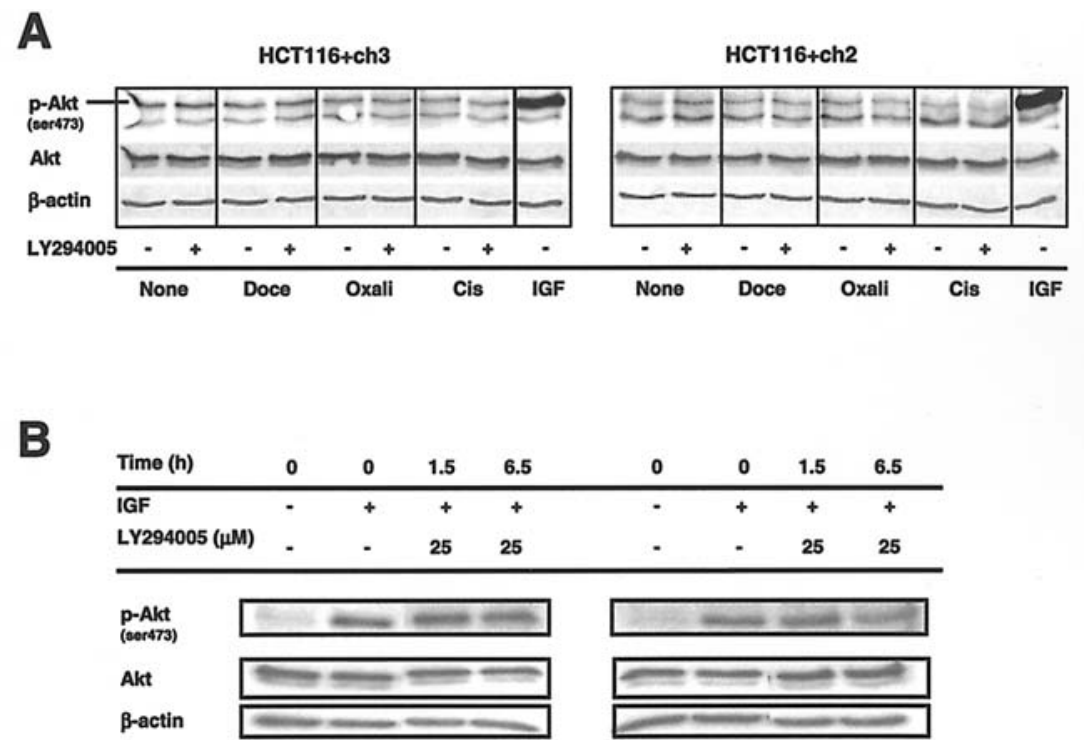

HCT116+ch3 HCT116+ch2
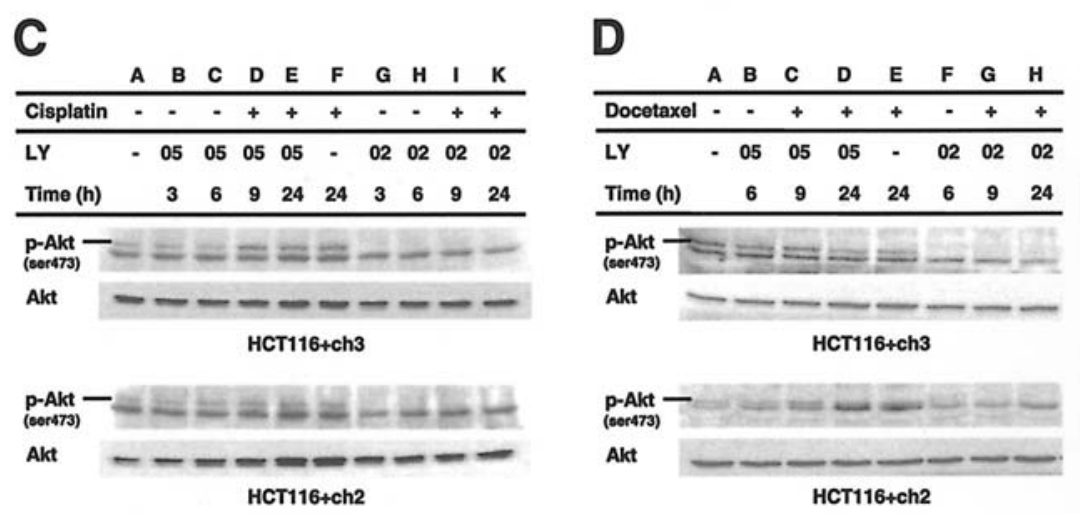

Figure 5. Effect of LY294005 or LY294002 on the level of phospho-Akt ${ }^{\text {ser473 }}$ as a function of the treatments. (A) Cells were incubated without (-) or with (+) $10 \mu \mathrm{M}$ LY294005 in the absence (none) or presence of $0.6 \mathrm{nM}$ Docetaxel (Doce), $5 \mu \mathrm{M}$ Oxaliplatin (Oxali), $5 \mu \mathrm{M}$ Cisplatin (Cis), or $20 \mathrm{ng} / \mathrm{ml}$ IGF. Levels of phospho-Akt ${ }^{\text {ser473 }}$, total Akt, and B-actin (sample loading control) are shown. (B) Cells were pre-treated without (-) or with $25 \mu \mathrm{M}$ LY294005 30 min prior to treatment without (-) or with $20 \mathrm{ng} / \mathrm{ml} \mathrm{IGF} \mathrm{(+),} \mathrm{and} \mathrm{were} \mathrm{analyzed} \mathrm{for} \mathrm{phospho-Akt}{ }^{\text {ser } 473}$, total Akt, and 3 -actin 1.5 or $6.5 \mathrm{~h}$ after IGF-treatment. (C and D)

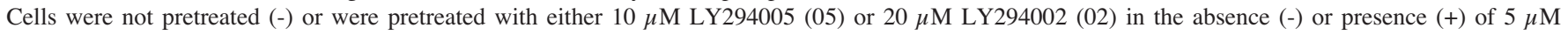
Cisplatin or $0.6 \mathrm{nM}$ Docetacel and analyzed as a function of time post the addition of LY294005 or LY294002. Levels of phospho-Akt ${ }^{\text {ser473 }}$ and total Akt are shown as a function of the treatments.

signaling pathway. Here we investigated whether selective inhibition of Akt by the compound LY294005 specifically reverses the Cisplatin resistance phenotype of tumor cells with MMR dysfunction. We show that i) LY294005 decreased the efficacy of platinum drugs against HCT116 tumor cells under experimental conditions it increased - as expected from the literature - that of Docetaxel, that ii) the extent of these LY294005-imposed decreases was reproducibly greater for the MLH1-proficient subline with Cisplatin and Lipoplatin and hence occurred with a preference for this subline, and that iii) neither the increases (Docetaxel) nor decreases (platinum drugs) in drug efficacy imposed by LY294005 were associated with concomitant alterations in the level of phosphorylated $\mathrm{Akt}^{\mathrm{ser} 473}$. Although not conclusive, these observations suggest a possible relationship between MMR-mediated DNA damage signaling and the Akt pathway and a possible novel property of Akt which, at least for platinum drugs and under certain circumstances, may aggravate rather than counteract cytotoxic cellular responses. They also suggest the potential of LY294005 to modulate drug sensitivity without affecting phosphorylation of $\mathrm{Akt}^{\mathrm{ser} 473}$.

Recent studies have shown that the PI3-kinase inhibitor LY294002 increased radio- and chemosensitivity with the concomitant reduction of phospho-Akt $(26,30)$. Likewise, Akt inhibition by LY294005 was insufficient to induce apoptosis by itself, but induced apoptosis in combination with a cytotoxic agent or radiation $(27,29,32)$. Chemosensitizing effects have also been reported for Akt-specific inhibitors such as A-443654 (32) and inositol pentakisphosphate (31). Our observations with LY294005 and Docetaxel are compatible with these studies.

With regard to these studies and to our observation that LY294005 sensitized HCT116 tumor cells to Docetaxel, we had hypothesized that LY294005 not only increases the efficacy of platinum compounds against HCT116 colorectal adenocarcinoma cells but also increases the cytotoxic effect of 
Cisplatin preferentially in (Cisplatin-resistant) MMR-deficient cells. Our hypothesis was based on the assumptions that i) MMR-responsive signaling represses the survival properties of Akt, thereby contributing to drug sensitivity in MMRproficient cells, and that ii) this repressing effect is abolished in the absence of MMR, giving rise to the drug resistance phenotype in MMR-deficient cells. We anticipated that the Akt inhibitor LY294005 overcomes the lack of this repressing effect of MMR on Akt signaling, with the net result that MMR-deficient cells should be rendered sensitive to Cisplatin.

However, our observations made with platinum compounds in general and with the cisplatinum compounds Cisplatin and Lipoplatin in particular were quite surprising and were not compatible with our hypotheses. On the one hand, our results indicate the potential of LY294005 to counteract platinum drug-induced cytostatic responses in HCT116 tumor cells, providing the cells with a growth advantage rather than preventing their growth. Our findings seem incompatible with the role for Akt as a positive regulator for cell growth and survival, and they may point to a possible novel property of Akt which, at least with platinum drugs and perhaps under certain circumstances, promotes cell death and/or antagonizes cytoprotective pathways. There are studies reporting Aktdependent induction of p21 and p53 after Cisplatin treatment, leading to growth arrest $(39,40)$. A possible explanation for our results may be that abrogation of this effect by LY294005 abolishes the growth arrest and renders the cells resistant to the cytotoxic effect of the platinum drugs.

On the other hand and perhaps more notable, our results may point to the existence of a relationship between MMRmediated cisplatinum DNA damage signaling and the Akt pathway. Such a relationship and the putative existence of common targets for MMR proteins and Akt have been suggested previously (16). However, our observations are incompatible with the initially hypothesized role for MMR in repressing the survival properties of Akt. Instead, it may be proposed that MMR-responsive signaling could antagonize the putative cell death-promoting property of Akt signaling. The molecular nature of the possible relationship and of the putative novel property of Akt is not known. Our hypothesis also predicted that Akt inhibition by LY294005 could specifically re-sensitize MLH1-deficient cells to cisplatinum drugs. This was, however, not the case. Noteworthy enough, our findings may not be unique to the LY294005 Akt inhibitor, because we made similar observations also with the PI3-kinase inhibitor LY294002. It seems that the observed effects of LY294005 and LY294002 are not limited to HCT116 tumor cells, since both inhibitors decreased the efficacy of Cisplatin also in HeLa cells (not shown). Our observations, however, were different from those in other studies reporting a Cisplatin sensitizing effect of LY294002 (28,30). As LY294005 increased the efficacy of the microtubule poison Docetaxel and did not affect that of the antimetabolite 6-thioguanine under experimental conditions it did with platinum drugs, it remains to be seen whether the potential of LY294005 and LY294002 to antagonize the efficacy of platinum compounds is restricted to this class of DNA cross-linking agents and hence may be DNA damage type-dependent, or whether it might also be observed with other classes of DNA modifying agents.
The observed lack of the abrogation of phospho-Akt $\mathrm{t}^{\mathrm{ser} 473}$ level by LY294005 with IGF and of the association of the LY294005-imposed changes in drug sensitivity (increase for Docetaxel and decrease for platinum drugs) with alterations in the level of phospho-Akt ${ }^{\text {ser473 }}$ may raise new issues regarding the function for LY294005 in this context. It is possible that LY294005 could affect targets other than Akt. It is also possible that Akt inhibition may not necessarily depend on the phosphorylation status of Akt, which seems to be compatible with a previous study reporting that Akt inhibition by A-443654 was associated with increased rather than decreased phosphoAkt, suggesting that the phospho-Akt level may not be a sufficient marker for Akt inhibition (33). Other studies have shown that Akt inhibition by LY294005 (31) and by PI3-kinase inhibitors such as LY294002 $(26,28,30)$ was associated with abrogation of Akt phosphorylation. In our study, the PI3-kinase inhibitor LY294002 completely abolished phosphorylation of $\mathrm{Akt}^{\mathrm{ser} 473}$, which was not the case with Akt inhibitor LY294005. It is unclear why both LY294005 and LY294002 similarly affected the efficacy of Cisplatin (decrease) and Docetaxel (increase), although their respective impact on abrogation of $\mathrm{Akt}^{\mathrm{ser} 473}$ was different, i.e. absent for LY294005 and present for LY294002.

Although not conclusive, our observations may point to a possible property of Akt, at least for platinum drugs and under certain circumstances, in aggravating the cytotoxic effect of these drugs, and to the existence of a functional relationship between MMR-mediated DNA damage response and the Akt signaling pathway. More detailed studies will be required to understand how LY294005 interferes with Akt signaling, why LY294005 affects the efficacy of taxanes differently from that of platinum drugs, and what the role for MMR might be in this context.

\section{Acknowledgements}

This work was supported by the Swiss National Science Foundation (grant no: 31-105300/1). The authors would like to thank Dr N. Lentze for technical assistance.

\section{References}

1. Modrich P and Lahue R: Mismatch repair in replication fidelity, genetic recombination, and cancer biology. Annu Rev Biochem 65: 101-133, 1996.

2. Jiricny J: Replication errors: cha(lle)nging the genome. EMBO J 17: 6427-6436, 1998.

3. Kolodner RD and Marsischky GT: Eukaryotic DNA mismatch repair. Curr Opin Genet Dev 9: 89-96, 1999.

4. Fishel R and Kolodner RD: Identification of mismatch repair genes and their role in the development of cancer. Curr Opin Genet Dev 5: 382-395, 1995.

5. Peltomaki P: Role of DNA mismatch repair defects in the pathogenesis of human cancer. J Clin Oncol 21: 1174-1179, 2003.

6. Hawn MT, Umar A, Carethers JM, Marra G, Kunkel TA, Boland CR and Koi M: Evidence for a connection between the mismatch repair system and the $\mathrm{G}_{2}$ cell cycle checkpoint. Cancer Res 55: 3721-3725, 1995 .

7. D'Atri S, Tentori L, Lacal PM, Graziani G, Pagani E, Benincasa E, Zambruno G, Bonmassar E and Jiricny J: Involvement of the mismatch repair system in temozolomide-induced apoptosis. Mol Pharmacol 54: 334-341, 1998

8. Branch P, Hampson R and Karran P: DNA mismatch binding defects, DNA damage tolerance, and mutator phenotypes in human colorectal carcinoma cell lines. Cancer Res 55: 2304-2309, 1995 
9. Fink D, Nebel S, Aebi S, Zheng H, Cenni B, Nehme A, Christen RD and Howell SB: The role of DNA mismatch repair in platinum drug resistance. Cancer Res 56: 4881-4886, 1996.

10. Swann PF, Waters TR, Moulton DC, Xu YZ, Zheng Q, Edwards M and Mace R: Role of postreplicative DNA mismatch repair in the cytotoxic action of thioguanine. Science 273: 1109-1111, 1996.

11. Fedier A, Fowst C, Tursi J, Geroni C, Haller U, Marchini S and Fink D: Brostallicin (PNU-166196) - a new DNA minor groove binder that retains sensitivity in DNA mismatch repair-deficient tumour cells. Br J Cancer 89: 1559-1565, 2003.

12. Fedier A, Schwarz VA, Walt H, Delli Carpini R, Haller U and Fink D: Resistance to topoisomerase poisons due to loss of DNA mismatch repair. Int J Cancer 93: 571-576, 2001.

13. Fedier A, Poyet C, Perucchini D, Boulikas T and Fink D: MLH1-deficient tumour cells are resistant to lipoplatin but retain sensitivity to lipoxal. Anticancer Drugs 17: 315-323, 2006.

14. Mackay HJ, Cameron D, Rahilly M, Mackean MJ, Paul J, Kaye SB and Brown R: Reduced MLH1 expression in breast tumors after primary chemotherapy predicts disease-free survival. J Clin Oncol 18: 87-93, 2000.

15. Samimi G, Fink D, Varki NM, Husain A, Hoskins WJ, Alberts DS and Howell SB: Analysis of MLH1 and MSH2 expression in ovarian cancer before and after platinum drug-based chemotherapy. Clin Cancer Res 6: 1415-1421, 2000.

16. Kandel ES, Skeen J, Majewski N, Di Cristofano A, Pandolfi PP, Feliciano CS, Gartel A and Hay N: Activation of Akt/protein kinase B overcomes a G(2)/m cell cycle checkpoint induced by DNA damage. Mol Cell Biol 22: 7831-7841, 2002.

17. Coffer PJ, Jin J and Woodgett JR: Protein kinase B (c-Akt): a multifunctional mediator of phosphatidylinositol 3-kinase activation. Biochem J 335: 1-13, 1998.

18. Vivanco I and Sawyers CL: The phosphatidylinositol 3-Kinase AKT pathway in human cancer. Nat Rev Cancer 2: 489-501, 2002.

19. Hanada M, Feng J and Hemmings BA: Structure, regulation and function of PKB/AKT - a major therapeutic target. Biochim Biophys Acta 1697: 3-16, 2004.

20. King FW, Skeen J, Hay N and Shtivelman E: Inhibition of Chk1 by activated PKB/Akt. Cell Cycle 3: 634-637, 2004

21. Hirose Y, Katayama M, Mirzoeva OK, Berger MS and Pieper RO: Akt activation suppresses Chk2-mediated, methylating agentinduced G2 arrest and protects from temozolomide-induced mitotic catastrophe and cellular senescence. Cancer Res 65: 4861-4869, 2005.

22. Datta SR, Dudek H, Tao X, Masters S, Fu H, Gotoh Y and Greenberg ME: Akt phosphorylation of BAD couples survival signals to the cell-intrinsic death machinery. Cell 91: 231-241, 1997.

23. Dan HC, Sun M, Kaneko S, Feldman RI, Nicosia SV, Wang HG, Tsang BK and Cheng JQ: Akt phosphorylation and stabilization of X-linked inhibitor of apoptosis protein (XIAP). J Biol Chem 279: 5405-5412, 2004.

24. Testa JR and Bellacosa A: AKT plays a central role in tumorigenesis. Proc Natl Acad Sci USA 98: 10983-10985, 2001.

25. Kim D, Dan HC, Park S, Yang L, Liu Q, Kaneko S, Ning J, He L, Yang H, Sun M, Nicosia SV and Cheng JQ: AKT/PKB signaling mechanisms in cancer and chemoresistance. Front Biosci 10: 975-987, 2005.

26. Nakamura JL, Karlsson A, Arvold ND, Gottschalk AR, Pieper RO, Stokoe D and Haas-Kogan DA: PKB/Akt mediates radiosensitization by the signaling inhibitor LY294002 in human malignant gliomas. J Neurooncol 71: 215-222, 2005.

27. Hu Y, Qiao L, Wang S, Rong SB, Meuillet EJ, Berggren M, Gallegos A, Powis G and Kozikowski AP: 3-(Hydroxymethyl)bearing phosphatidylinositol ether lipid analogues and carbonate surrogates block PI3-K, Akt, and cancer cell growth. J Med Chem 43: 3045-3051, 2000.
28. Tsurutani J, West KA, Sayyah J, Gills JJ and Dennis PA Inhibition of the phosphatidylinositol 3-kinase/Akt/mammalian target of rapamycin pathway but not the MEK/ERK pathway attenuates laminin-mediated small cell lung cancer cellular survival and resistance to imatinib mesylate or chemotherapy. Cancer Res 65: 8423-8432, 2005.

29. Martelli AM, Tazzari PL, Tabellini G, Bortul R, Billi AM, Manzoli L, Ruggeri A, Conte R and Cocco L: A new selective AKT pharmacological inhibitor reduces resistance to chemotherapeutic drugs, TRAIL, all-trans-retinoic acid, and ionizing radiation of human leukemia cells. Leukemia 17: 1794-1805, 2003.

30. Viniegra JG, Losa JH, Sanchez-Arevalo VJ, Parada Cobo C, Soria VM, Ramon y Cajal S and Sanchez-Prieto R: Modulation of PI3K/Akt pathway by E1a mediates sensitivity to cisplatin. Oncogene 21: 7131-7136, 2002.

31. Maffucci T, Piccolo E, Cumashi A, Iezzi M, Riley AM, Saiardi A, Godage HY, Rossi C, Broggini M, Iacobelli S, Potter BV, Innocenti $\mathrm{P}$ and Falasca M: Inhibition of the phosphatidylinositol 3-kinase/Akt pathway by inositol pentakisphosphate results in antiangiogenic and antitumor effects. Cancer Res 65: 8339-8349, 2005.

32. Hu H, Jiang C, Li G and Lu J: PKB/AKT and ERK regulation of caspase-mediated apoptosis by methylseleninic acid in LNCaP prostate cancer cells. Carcinogenesis 26: 1374-1381, 2005

33. Luo Y, Shoemaker AR, Liu X, Woods KW, Thomas SA, De Jong R, Han EK, Li T, Stoll VS, Powlas JA, Oleksijew A, Mitten MJ, Shi Y, Guan R, McGonigal TP, Klinghofer V, Johnson EF, Leverson JD, Bouska JJ, Mamo M, Smith RA, Gramling-Evans EE, Zinker BA, Mika AK, Nguyen PT, Oltersdorf T, Rosenberg SH, Li Q and Giranda VL: Potent and selective inhibitors of Akt kinases slow the progress of tumors in vivo. Mol Cancer Ther 4: 977-986, 2005.

34. Viniegra JG, Martinez N, Modirassari P, Losa JH, Parada Cobo C, Lobo VJ, Luquero CI, Alvarez-Vallina L, Ramon y Cajal S, Rojas JM and Sanchez-Prieto R: Full activation of PKB/Akt in response to insulin or ionizing radiation is mediated through ATM. J Biol Chem 280: 4029-4036, 2005.

35. Suzuki A, Kusakai G, Kishimoto A, Lu J, Ogura T, Lavin MF and Esumi H: Identification of a novel protein kinase mediating Akt survival signaling to the ATM protein. J Biol Chem 278: 48-53, 2003

36. Altiok S, Batt D, Altiok N, Papautsky A, Downward J, Roberts TM and Avraham H: Heregulin induces phosphorylation of BRCA1 through phosphatidylinositol 3-Kinase/AKT in breast cancer cells. J Biol Chem 274: 32274-32278, 1999.

37. Wang Y, Cortez D, Yazdi P, Neff N, Elledge SJ and Qin J: BASC, a super complex of BRCA1-associated proteins involved in the recognition and repair of aberrant DNA structures. Genes Dev 14: 927-939, 2000

38. Boulikas T: Low toxicity and anticancer activity of a novel liposomal Cisplatin (Lipoplatin) in mouse xenografts. Oncol Rep 12: 3-12, 2004.

39. Mitsuuchi Y, Johnson SW, Selvakumaran M, Williams SJ, Hamilton TC and Testa JR: The phosphatidylinositol 3kinase/AKT signal transduction pathway plays a critical role in the expression of $\mathrm{p} 21 \mathrm{WAF} 1 / \mathrm{CIP} 1 / \mathrm{SDI} 1$ induced by cisplatin and paclitaxel. Cancer Res 60: 5390-5394, 2000.

40. Miyauchi H, Minamino T, Tateno K, Kunieda T, Toko H and Komuro I: Akt negatively regulates the in vitro lifespan of human endothelial cells via a p53/p21-dependent pathway. EMBO J 23: 212-220, 2004. 